\title{
Effect of Shape of Magnet on the Machining of Workpiece
}

\author{
K. Srinivas, Q. Murtaza, A.K Aggarwal
}

\begin{abstract}
Viscoelastic Magnetic Abrasive finishing has achieved a wide popularity in the field of finishing as the process is capable of finishing Non-ferrous material along with the ferrous material. With the development in technology the quality and surface finish attract more attention as compare to the machining perfection and dimensional accuracy. The present paper consists of comparison of four type of magnet available in the market and one of them as self-proposed electromagnet. A single aluminium hollow cylindrical workpiece of outer diameter as $10 \mathrm{~mm}$ and the inner diameter as $8 \mathrm{~mm}$ is finished by taking the magnet one by one whose dimensions are made according to the available literature and market and are placed as two magnetic poles with 180 degrees apparats. Ansys Maxwell 16 software was used to predict the value of maximum magnetic field on the workpiece due to the shape of the magnet and it was found that the maximum magnetic field was produced by fan shape magnet of about 0.6 Tesla. From the idea of magnetic field intensity of the workpiece, the machining ability of the magnet in viscoelastic magnetic field can be produced.
\end{abstract}

Keywords: Viscoelastic Magnetic Abrasive finishing, Finishing, Electromagnet.

\section{INTRODUCTION}

Magnetic abrasive finishing is an effective machining process in which magnetic field is used to machine the target workpiece for machining. In conventional abrasive machining process both the workpiece and the target surface are polarised and due to the interplay of magnetic field, a Brush of abrasive is formed which results in the effective machining of the workpiece. This effective concept of machining got the eyeball of many scholar and initiated a large number of researches in this field like Kang and Yamaguchi [1] developed a multipole dip- system for the machining of internal surface of the capillary tube. The researchers highlighted the difficulties faced in machining of the capillary tube mainly in controlling the magnetics abrasive in MAF process, the researchers suggested that the multipole system with alternate magnetics and nonmagnetic region simultaneously applied on the workpiece which results in the effective machining of the long capillary tube. This process has allowed the application of MAF on long Capillary tube as well which was earlier confined to the short length tube only. The final conclusion of the researcher was

Revised Version Manuscript Received on 10, September 2019.

K. Srinivas, Assistant Professor Delhi Technological University New Deli, India.(Email: srinivaskrovvidi@dtu.ac.in)

Q. Murtaza, Professor Delhi Technological University New Delhi, India. India. surfaces of the workpiece by influencing the carbonyl particles and directing them towards the wall of the

that the double pole system finishes the workpiece in half a time as compare to the single pole system. V.K Jain [2] explained various non-traditional machining process which are used in modern times which involves the use of abrasive and the media to support it. The various process which he discussed in its paper was Abrasive Flow Finishing (AFF), Magnetic Abrasive Finishing (MAF), Magnetorheological Finishing, Magnetorheological Abrasive Flow Finishing, Elastic Emission Machining (EEM) and Magnetic Float Polishing. he laid attention on the magnetic abrasive finishing process and gave some important conclusion like involvement of slot in the magnet over non machining zone and the use of pulse Dc supply rather than smooth DC supply in the system. Wani et al. [4] gave stress on Magnetic Abrasive flow finishing which is a process made by combining the advantage of both abrasive flow machining and the magnetic abrasive finishing process, and used finite element method to find distribution of magnetic potential in the magnetic brush. Through this distribution the researcher evaluated machining pressure, surface finishing and material removal. After the successful evaluation of the results the researcher claims that these results are similar to those present in the literature. Kala et al. [4] developed a setup which aims to catch the force signature for double disc magnetic abrasive finishing process and the conventional abrasive magnetic abrasive finishing process and by these properties the researchers aims to understand the working of the flexible magnetic abrasive brush. Thus, to do this the researchers performed the magnetic abrasive finishing operation in single as well as double disc and finally understood the property of flexible magnetic abrasive brush behaviour from it. Wu et al [5] developed a model of magnetic abrasive finishing process and analysed its properties based on the rotational speed, cutting fluid and the magnetic pole and current frequency and its effect on change in material removal and surface finish are invested. The researchers finally claimed that the neat cutting oil is required for processing which results in higher material removal and finer finish. They also concluded that finishing is better with the increasing in rotational speed and as the current frequency increases the angle variation of the particle increases and if frequency is low the surface requirement is up to few nanometres. Rathod et al. [6] correlated the difference shape factor with wide range of particle size. The researcher made use of slurry abrasion tester with silica sand slurry and commented on the effect of sliding distance slurry abrasion volume, where they argued that volume loss is proportional 
to the sliding distance. They also studied the effect of micro ploughing and cutting. Srivastav et al. [7] simulated a model of electrochemical magnetic abrasive finishing process in which the researchers used stainless steel workpiece whose radius was ten $\mathrm{mm}$ and thickness was $2.5 \mathrm{~mm}$. the researchers used chromium oxide as the abrasive particle whose average diameter was 387 micrometres, the intensity of magnetic field was around 1 tesla and the rotational speed of tool was taken as $5835 \mathrm{rpm}$ and its diameter was $6 \mathrm{~mm}$. the electrolyte used for the experiment was brine solution with twenty percent concentration. The researchers study the thermal model in both steady and transient model. Srivastav and Amit [8] did the thermal analysis of work-brush interface and along the workpiece depth in magnetitic abrasive finishing process with the help of Ansys R15 software in both steady and thermal state. The value of magnetic field intensity given to 6 mm diameter tool was 1 tesla. The researcher used chromium oxide abrasives of about 387 micrometre Diameter. The workpiece has a radius of $10 \mathrm{~mm}$ and $2.5 \mathrm{~mm}$ thickness and the material of the workpiece was silicon nitride. Singh et al. [9] used Taguachi L orthogonal array to design an experiment which could measure surface temperature of the finishing surface of mild steel using MAF process. The researchers used current, working gap, Rotational speed and abrasive weight as its important process parameters. After the Taguachi optimization the Fuzzy logic is used by researcher through which prediction of surface temperature is and also it is validated. Jaiswal et al. [10] made FEM model of MAF and investigated the distribution of magnetic force on the workpiece. The researcher also made a theoretical model for computing the material removal and surface roughness. For this the workpiece was considered of uniform surface without statically distribution. Numerical experiments were also computed which were validated later on through literature. Yan et all [11] performed the experiment on MA genetic abrasive finishing based on a simulated model made by FEM model electromagnetic inductor. He then compared the simulated model with the actual electromagnetic inductor and predicted a marginal error of only 7 percent. Li et all [12] developed a finishing setup having new media for finishing the rotatory surface ANSYS Maxwell was used to determine the optimum angle between the poles and the mathematical model was made to compute the MRR in which pressure and velocity was computed based on arced wear model. The MRR computation model was made which predicted MRR as a function of pole rotational speed, Magnetic flux density, cam rotational speed and the ferromagnetic and abrasive particles' diameter. Li et all [13] proposed a new viscoelastic magnetic Abrasive based on analysis of field characteristic and existing finishing process. They also discussed the motion locus of the abrasives. The present scenario, abrasive flowing finishing, grinding, abrasive brush deburring, magnetorheological finishing, manual deburring etc. are broad name in term of finishing but still they possess some limitations in finishing quality and efficiency. Shimamura et al. [14], [15] modelled and tested that magnetic abrasive particles are subjected to the pressure for machining and that pressure is a function of magnetic flux density, number of abrasive particles and the permeability of abrasive medium. Fox et al [16] observed that the magnetic force (function of the volume and magnetic susceptibility of the ferrous

particles in the magnetic field, magnetic field intensity and the gradients at the finishing area). Srinivas et al. [17] studied the distribution of the magnetic field intensity along with the computation of torque and force and related flow parameters of Viscoelastic magnetic Abrasive finishing process with magnetic field intensity. The Researchers used Ansys Maxwell and Ansys Fluent in order to find co relation between the flow parameters and magnetic field intensity and commented on the successful application of the proposed viscoelastic media. The researchers also plotted the graph between the magnetic field intensity and the current which is applied on the single pole electromagnet to produce the desired magnetic field for machining in the magnetic abrasive finishing process. Srinivas and Anant [18] studied the pressure variation in abrasive flow machining process with the help of a simulated model made on Ansys Fluent and commented on the effect of pressure variation in the machining operation. They used viscous media similar to that used in viscoelastic magnetic abrasive finishing with the difference that in later process there is an addition of carbonyl particles which gets activated by magnetic field. The researcher computed pressure difference between $40 \mathrm{MPa}$ and $20 \mathrm{MPa}$ and the value of pressure so obtained was validated with the help of literature review. Ali et al. [19] reviewed various researches on AFM which uses a viscoelastic media and semisolid media which can support various abrasive based finishing process including both magnetic and non-magnetic based finishing process. Bhardwaj et al [20] compiled the researches which contained the use of viscoelastic media in various hybrids of abrasive flow machining process, the researchers also commented on the tooling and fixturing in various hybrids of AFM and the compatibility of the viscoelastic media with the hybrid process. So, it can be seen that Viscoelastic Magnetic Abrasive Finishing has a vital role in Finishing department and must be consider for effective machining operation.

\section{METHODOLOGY}

As mentioned above the aim of the paper was to identify the machining effect due to the shape of the magnet. For this the Ansys Maxwell 16 was used in which simulations of different shape of the magnet was done and the magnetic Field intensity was calculated.The setup is so designed that it consist of a hollow work piece whose internal surface is to be machined by the viscoelastic fluid which is made to reciprocate inside the work piece by providing the pressure difference in presence of magnetic field produced by providing the current in the copper coils, which are acting like the magnetic poles. The resultant combination of the force due to magnetic field and the force due to Pressure variation would produce an effective machining. The viscoelastic media so produced consist of Ferromagnetic particles around which 4 abrasive particles are aligned due to volume their ratio, which is $1: 4$ by volume. Alumina is the material of abrasive particle and the ferromagnetic particle is iron powder, which is commercially known as Carbonyl Iron Powder. The workpiece material aluminium and the 
magnetic poles are made of copper. CREO 3.0 is used for making the initial model, which is later imported to Ansys Maxwell for analysis. The Shape of the mesh element is Hexagonal and the total number of elements are $3 \times 10^{3}$. After several trials it is concluded that the result obtained is independent of the mesh size. The electromagnet which is used is designed on CREO 3.0 which is of hollow rectangular copper block of copper material which is illustrated in figure 2. The magnet so chosen, follows the faraday's law of electromagnetic induction, it produces a magnetic field around the magnet, when the current is passed through it .Using Boolean operation on the Maxwell 16 software, a surface is created and a current of $100 \mathrm{~A}$ is allowed in the clockwise direction on each surface of the magnet which results in the directional magnetic field towards the work piece thus attracting more fluid particle towards the work piece, which aids in effective machining with combination of applied pressure and magnetic field. So, the general idea is to get the combination of the effective machining by the result combination of extrusion Pressure and the magnetic field intensity.

\section{RESULTS AND DISCUSSION}

For the electromagnet it was observed that the maximum and smoother finish is achieved at the region of the work piece which is in front of the hollow part of the electromagnet. This application of the electromagnet is used in the motor also which are based on electromagnet. From the simulation results it can be observed that For The model made when the two magnets are arranged at an angle of 180 degree with each other, on applying the clockwise current of $100 \mathrm{~A}$ on the surface (made by Boolean operation) of each magnet a pattern is produced with maximum magnetic field intensity of $4.0064 \times 10^{-2} \mathrm{~T}$. The figure 1 Shows the distribution of magnetic field when the arrangement of poles is 180 degree with each other. The next simulation was done for the Fan shaped magnet which was made whose inner diameter of the magnetic pole is $32 \mathrm{~mm}$, outer diameter of the magnetic pole is $126 \mathrm{~mm}$ and the thickness of the pole is 30 $\mathrm{mm}$. For this magnet also the orientation was 180 degrees angle between the pole and the simulated results are for Figure 3 the Force on abrasives is $0.22427 \mathrm{~N}$ and that on iron particle is $0.18769 \mathrm{~N}$ and the maximum value of magnetic field is $0.6205 \mathrm{~T}$. The next test was done on the Arc shaped magnet (Figure 4) which was made according to the dimensions which are commercially available in the market which is outer radius as $15.8 \mathrm{~mm}$, inner radius as $12.8 \mathrm{~mm}$ length of outer arc as $24.8 \mathrm{~mm}$ and the thickness is $41 \mathrm{~mm}$. the cylindrical magnet is also placed with the 180 degrees apart and the obtained maximum value of magnetic field intensity is $0.77 \mathrm{~T}$. Similar conditions is given for the ring shaped magnet and it shows the $0.33 \mathrm{~T}$ of the magnetic field.

\section{CONCLUSIONS}

- Viscoelastic Magnetic Abrasive finishing is effective mode to achieve Nano finishing

- Among the four-magnet used Arc shaped magnet shows the maximum magnetic field intensity.

Among the four-magnet used the Ring Shaped magnet gave the minimum value of the magnetic field intensity.

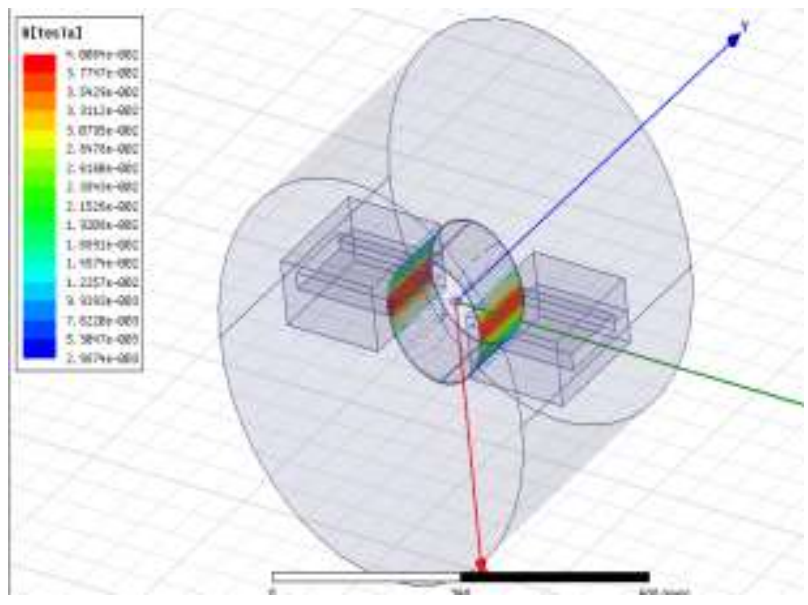

Figure 1 Magnetic Field produced By Electromagnet
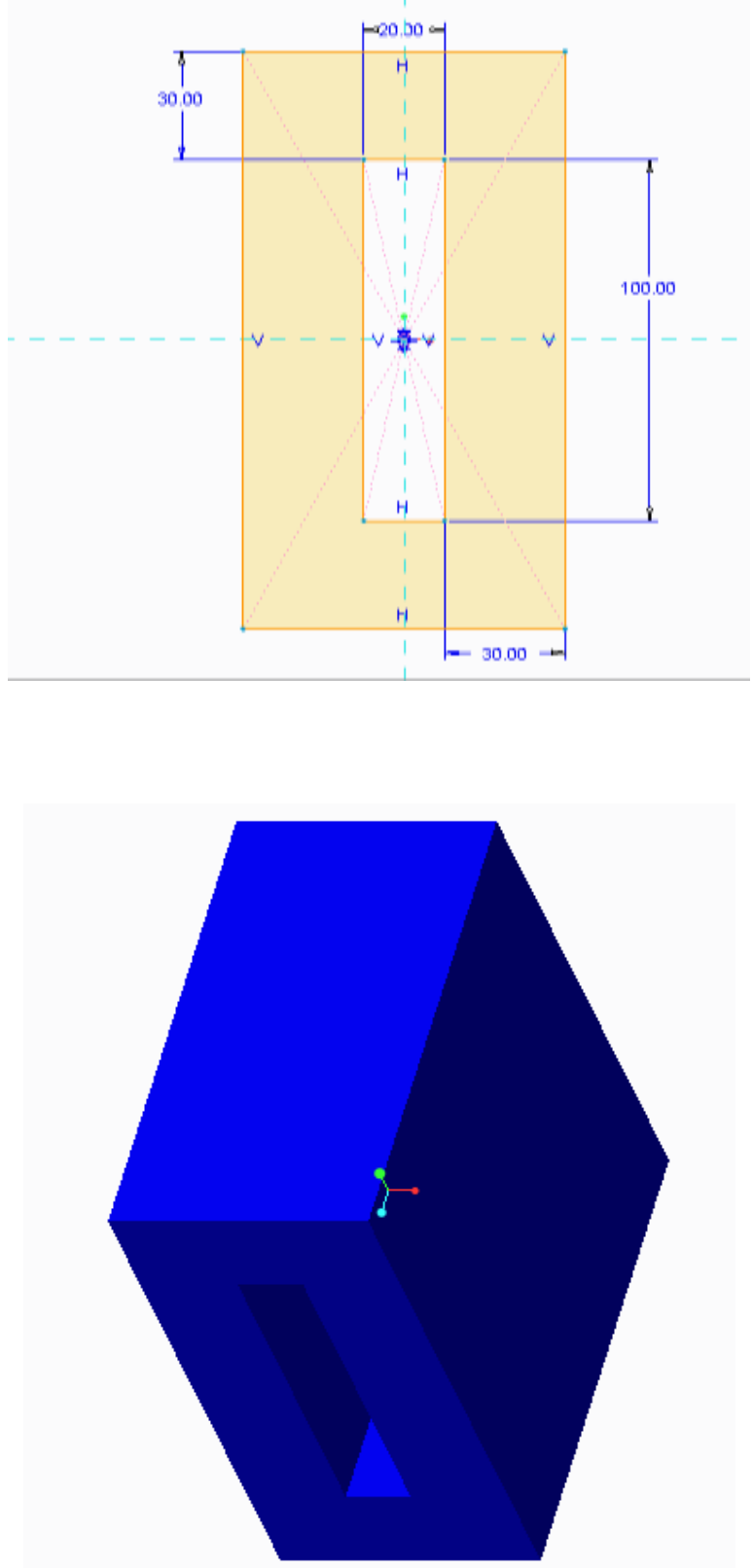

Figure 2 Dimensions of Proposed Electro Magnet

Published By: Blue Eyes Intelligence Engineering \& Sciences Publication 


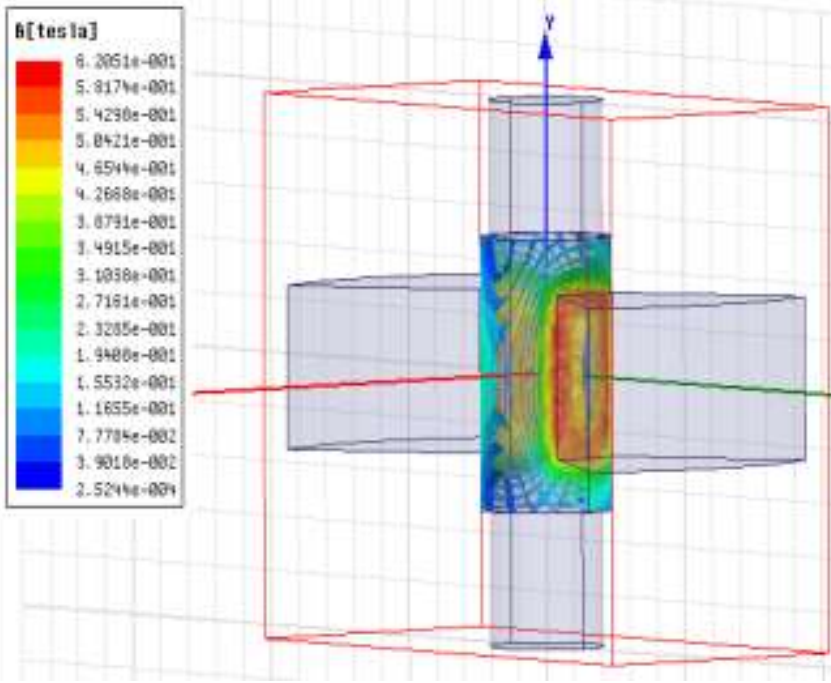

Figure 3 Magnetic Field Intensity for Fan Shaped Permanent Magnet

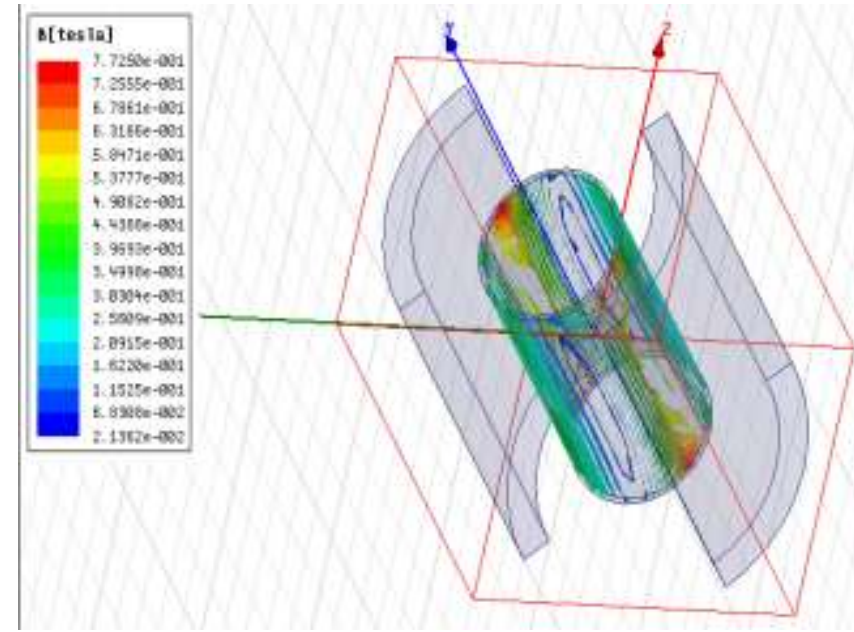

Figure 4 Magnetic Field Intensity for arc Shaped Permanent Magnet

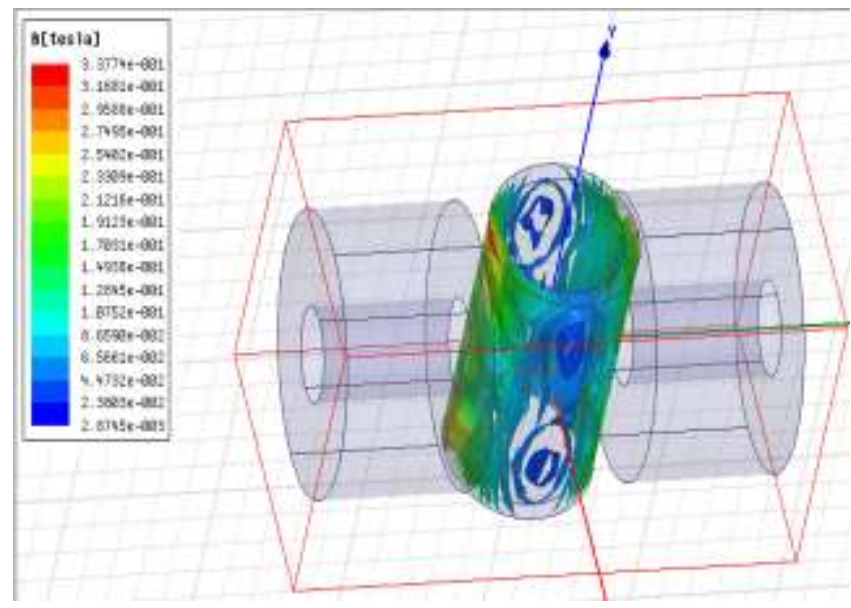

Figure 5 Magnetic Field Intensity for Ring Shaped Permanent Magnet

\section{REFERENCES}

1. Kang, Junmo., Yamaguchi, Hitomi: Internal finishing of capillary tubes by magnetic abrasive finishing using a multiple pole-tip system, Precision Engineering 36 (2012) 510-516, 2012.

2. Jain, V. K: Magnetic field assisted abrasive based micro-/nano- finishing, Journal of Materials Processing Technology 209 (2009) 6022-6038, 2009.

3. Wani, Amit. M., Yadava, Vinod., Khatri, Atul: Simulation for the prediction of surface roughness in magnetic abrasive flow finishing (MAFF), Journal of Materials Processing Technology 190 (2007) 282-290.

4. Kala, Prateek., Pandey, Pulak. M., Verma, Girish. C., Sharma, Varun: Understanding flexible abrasive brush behaviour for double disk magnetic abrasive finishing based on force signature, Journal of Manufacturing Processes 28 (2017) 442-448, 2017.

5. Wu Jinzhong., Zou, Yanhua., Sugiyama, Hitoshi: Study on finishing characteristics of magnetic abrasive finishing process using low-frequency alternating magnetic field, Int J Adv Manufacturing Technology 85:585- 594 DOI 10.1007/s00170-015-7962-9, (2016).

6. Rathod, avishkar., sapate, s. G., khatirkar, r. K: shape factor analysis of abrasive particles used in slurry abrasion testing, international journal of mechanical and industrial engineering (ijmie) issn no. $2231-6477$, vol-2, iss-4, 2012.

7. Srivastava, Ankit. Kumar., komma, V.R: Finite Element Modelling of Electrochemical Magnetic Abrasive Finishing, Imperial Journal of Interdisciplinary Research (IJIR), Vol- 2, Issue-12, 2016, ISSN: 2454-1362.

8. Srivastava, Ankit. Kumar., Katiyar. Amit.: Modelling and Simulation of Magnetic Abrasive Finishing for Thermal Analysis, Imperial Journal of Interdisciplinary Research (IJIR) Vol-2, Issue-12, 2016 ISSN: 2454-1362.

9. Singh, Rajneesh. Kumar., Singh, D.K., Gangwar. Swati.: Fuzzy logic optimization of process parameters affecting surface temperature in MAF, IOP Conf. Series: Materials Science and Engineering $404 \quad$ (2018) 012017 doi:10.1088/1757-899X/404/1/012017.

10. Jaiswal, S.C., Jain, V. K., Dixit, P.M.: Modelling and simulation of magnetic abrasive finishing process, Int $\mathbf{J}$ Adv Manufacturing Technology (2005) 26: 477-490, DOI 10.1007/s00170-004-2180.

11. Yan, Q. S., Qiu, T.X., Gao, W.Q., Meng, L: Simulation and Experimental Analysis of Electromagnetic Inductor for Magnetic Abrasive Finishing, Key Engineering Materials Vols. 389-390 (2009) pp 193-198 Online: 2008-09-26 (C) (2009) Trans Tech Publications, Switzerland, doi: 10.4028/www.scientific.net/KEM.389390.193.

12. Li, Wenhui., Li Xiuhong., Yang, Shengqiang., Li, Weidong: A newly developed media for magenetic abrasive finishing process: Material removal behaviour and finishing performance, Journal of Materials Processing Tech 260(2018) 20-29.

13. Li, Xiuhong., Li, Wenhui., Yang, Shengqiang.: Effect of coupling Agent on Interfecial Bonding Properties of Visecoelastic Magnetic Abrasive Tools and Finishing Performance, ISSN 1473-804 online, 1473-8031 print, DOI 10.5013/IJSSST.a.17.28.17.

14. Shinmura T, Takazawa K, Hatano E, Matsunaga M. (1990) Study on magnetic abrasive finishing. Ann CIRP, 39(1): 325-328.

15. Shinmura T., Takazawa K., Hatano E., Aizawa T. (1985) Study on magnetic abrasive process-process principles 
and finishing possibility, Bull. Japan Soc. Prec. Eng. 19(1) $54-55$.

16. .Kim JD., Choi MS. (1995) Simulation for the prediction of surface accuracy in magnetic abrasive machining. J Mater Process Tech 53:630-642.

17. Srinivas. K., Murtaza. Q., Aggarwal. A.K.: Variation of Flow parameters and Magnetic Flux in Viscoelastic Magnetic Abrasive Finishing process, ISSN 0973-4562 Volume 14, Number 8, pp. 1940-1946, (2019).

18. Srinivas. K., Bhardwaj, Anant.: Pressure variation in Abrasive Flow machining: Modelling and simulation, 3rd international conference on Advanced Production and Industrial Engineering (ICAPIE'18), Paper ID ICAPIE-2018-PE MT 218.

19. Ali, Parvesh., Ranganath, M.S., Walia, R.S., Murtaza, Q.: Various developments in Abrasive flow machining process: A Review, 3rd international conference on Advanced Production and Industrial Engineering (ICAPIE'18), Paper ID ICAPIE-2018- PE MT 215.

20. Bhardwaj, Anant., Ali, Parvesh., Walia, R. S., Murtaza, Qassim., Pandey, S. M.: Development of Hybrid Forms of Abrasive Flow Machining Process: A Review, Advances in Industrial and Production Engineering pp 41-67, Part of the Lecture Notes in Mechanical Engineering book series (LNME), conference paper (2019). 